\title{
The DELPHI Microvertex Detector
}

\author{
M. Caccia
}

\begin{abstract}
The main characteristics of the DELPHI Microvertex Detector are presented. The performance in terms of impact parameter resolution, association efficiency and ambiguity is evaluated after two years of data taking at LEP.
\end{abstract}

Presented in the XXVI International Conference in High Energy Physics Dallas, Texas, USA, August 5-12, 1992 



\section{INTRODUCTION}

The DELPHI Micro Vertex Detector (VD) has been installed since 1990 and it has been upgraded in 1991. About 410,000 hadronic $Z^{0}$ events have been collected over the two years; published results on heavy quarks and tau lepton physics show the importance of the VD precision measurements ${ }^{1}$. The detector layout is described here and its performance is reported. A more detailed description can be found elsewhere ${ }^{2}$.

\section{GENERAL LAYOUT AND DETECTOR CHARACTERISTICS}

A schematic view of the Silicon Vertex Detector is shown in Figure 1. It consists of three shells of Si microstrip detectors at average radii of $6.3 \mathrm{~cm}$ (Closer layer), $9 \mathrm{~cm}$ (Inner layer) and $11 \mathrm{~cm}$ (Outer layer). The Closer layer (installed in the 1991 upgrade) is $20.8 \mathrm{~cm}$ long while the length of the other two layers is $23.6 \mathrm{~cm}$. The strips are parallel to the beam direction and the transverse coordinate is measured. Each layer has 24 modules with about $10^{\circ}$ azimuthal overlap. The modularity has been chosen to avoid the intrinsic resolution degradation by inclined tracks. The overlap region was designed to improve the relative alignment of neighbouring modules.

Each module consists of four detectors. The pair in the same hemisphere are wire bonded in series and read out at the corresponding end by custom designed CMOS VLSI chips ${ }^{3}$. The average detector thickness is $280 \mu \mathrm{m}$; the strip pitch is $25 \mu \mathrm{m}$ but only every second strip is read out to match the readout chip input pitch. Closer, Inner and Outer layer detectors have respectively 384, 512 and 640 readout channels per side; altogether the number of readout strips is 73,728 . The main design characteristics of the detectors are $\mathrm{AC}$ coupling between the $p^{+}$strips
Table 1. Si detector electrical characteristics

\begin{tabular}{lcc}
\hline \hline Resistivity & $3-5$ & $k \Omega \mathrm{cm}$ \\
Depletion Voltage & $60-100$ & $\mathrm{~V}$ \\
Coupling Capacitance & $\geq 7$ & $\mathrm{pF} / \mathrm{cm}$ \\
Detector Capacitance & 1.1 & $\mathrm{pF} / \mathrm{cm}$ \\
\hline \hline
\end{tabular}

and the readout metal line, achieved through Silicon dioxide deposition, and biasing by integrated polysilicon resistors ${ }^{4}$. The main electrical characteristics of the detectors are summarized in Table 1.

Two parameters characterizing the detector are the signal over noise ratio and the number of strips in a cluster associated to a charged particle. A cluster is defined as the row of adjacent strips collecting the charge carriers generated by an ionizing particle. A strip is included in a cluster if the ratio of its Pulse Height (PH) to its Noise (N) exceeds $20 \%$ of the highest $\mathrm{PH} / \mathrm{N}$ value in the cluster itself. A cluster is then accepted if the sum of the $\mathrm{PH} / \mathrm{N}$ of the individual channels is larger than 6 and if there is at least one channel with a $\mathrm{PH} / \mathrm{N}$ larger than 3. The distribution of the cluster pulse height normalized to the one-strip noise is shown in Figure 2.

The most probable value of $\mathrm{PH} / \mathrm{N}$ for hits associated to a track is 15 and it can be understood in terms of three different contributions. The most important one comes from the amplifier noise; the single amplifier Equivalent Noise Charge (ENC) can be written as

$$
E N C_{\text {amplifier }}=670 e^{-}+55 \frac{e^{-}}{\mathrm{pF}} C_{D}
$$

where $C_{D}$ is the detector capacitance. Taking into account the values in Table 1 and the anticorrelation term in the actual configuration, the amplifier noise for two strip clusters is about 1300 r.m.s. electrons.

The shot noise contribution is ${ }^{5}$ 


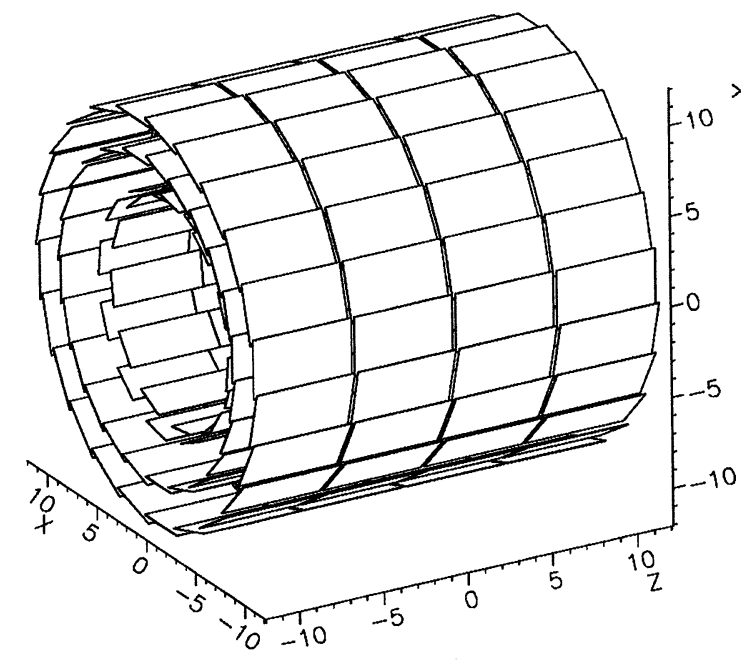

Figure 1. Schematic layout of the DELPHI Microvertex Detector.

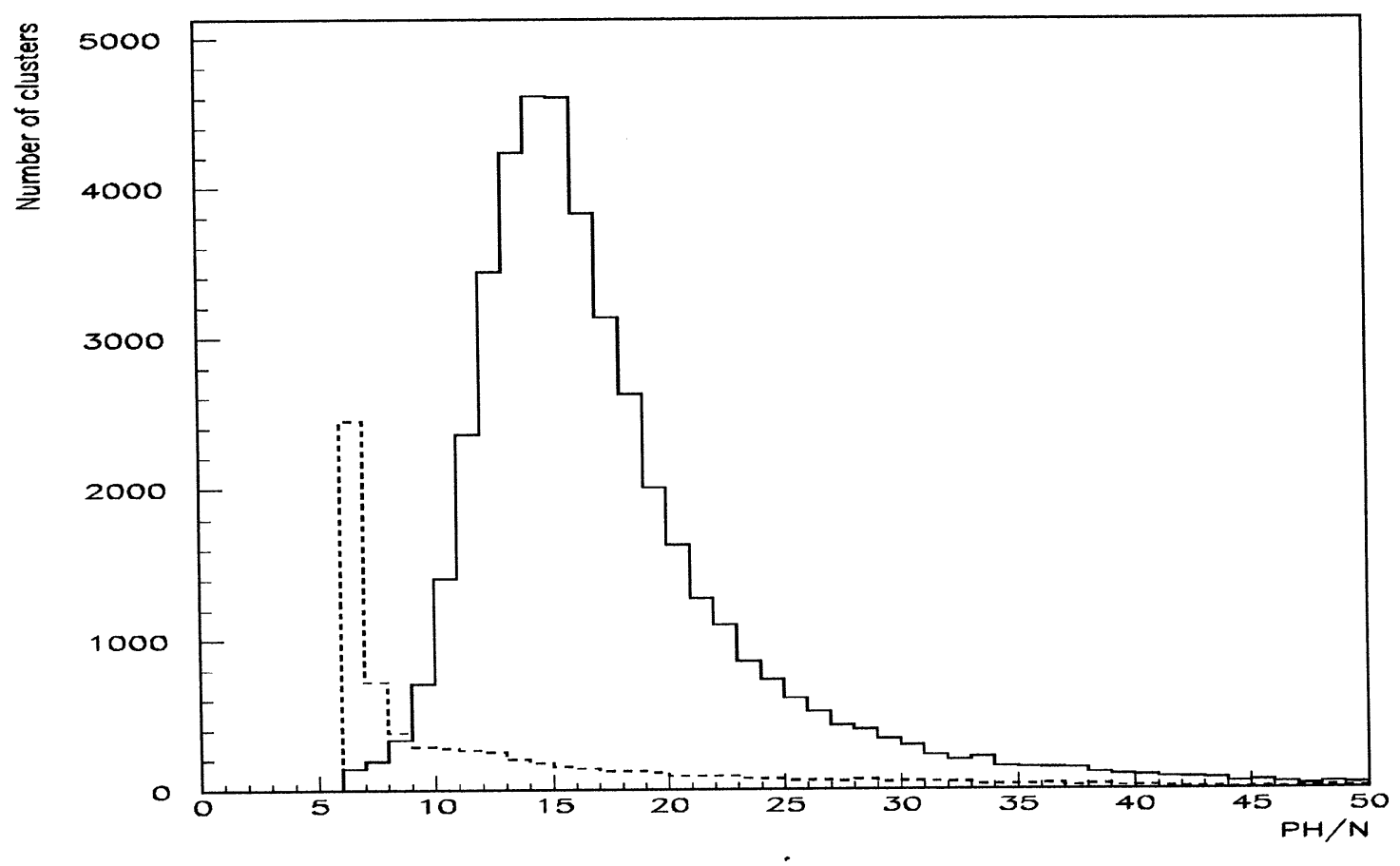

Figure 2. $\mathrm{PH} / \mathrm{N}$ for clusters with (full-line) and without (dashed-line) a track associated to them for the Inner layer modules. The PH/N has been normalized to the minimum track length in traversing the silicon. 


$$
E N C_{I_{\text {leakage }}} \simeq 100 \sqrt{I_{\text {leakage }}(\mathrm{nA})} e^{-}=200 e^{-}
$$

since $<I_{\text {leakage }}>=4 \mathrm{nA} /$ strip.

The thermal noise coming from the bias resistor is ${ }^{5}$

$$
E N C_{R_{b i a s}} \simeq \frac{760 e^{-}}{\sqrt{R_{b i a s}(M \Omega)}}=380 e^{-}
$$

when $<R_{\text {bias }}>=4 M \Omega$

The most probable energy loss corresponds to about 20,000 hole-electron pairs. Normalizing this value to the sum in quadrature of the three noise sources, a value in fair agreement with the measured one is obtained.

The information on the cluster width as a function of the track incidence angle is shown in Figure 3. The highest probability for one strip clusters does not correspond to a normal incidence angle. This can be understood in terms of the transverse shift of the charge carrier distribution induced by a Lorentz force term $^{6}$ associated to the DELPHI magnetic field. Because of the interleaved strip between two readout ones and the associated charge division, the fraction of multi strip clusters is always higher than $70 \%$. The detector acceptance and the strip pitch mainly define the fraction of 2 strip clusters while delta rays and two-track separation have to be taken into account as a source of larger clusters.

Silicon strip detectors with similar characteristics guarantee 6-7 $\mu \mathrm{m}$ intrinsic precision ${ }^{2,7}$ but the effective resolution in the VD design is also determined by other factors.

A severe limitation may come from the uncertainty in the relative position of the modules. In order to minimize it, the mechanical structure is surveyed before the installation $^{8}$ and an off-line analysis of reconstructed tracks ${ }^{9}$ refines the results. The detector stability is monitored during data taking with laser spots shining on the Outer-layer detectors and with capacitive probes mounted on the VD mechanical structure ${ }^{10}$.

We achieve 7-10 $\mu \mathrm{m}$ survey precision on the radial coordinate and $4-5 \mu \mathrm{m}$ alignment precision in the transverse coordinate, resulting into an effective measured resolution of $8 \mu \mathrm{m}$ per point (see below). No movements in excess of $10 \mu \mathrm{m}$ were recorded by the position monitor systems during the data taking periods.

\section{DETECTOR PERFORMANCE}

The VD performance can be summarized by the reconstruction quality on a single track, the detector and association efficiency, and by the ambiguity level in the pattern recognition.

\section{Spatial Resolution}

The $Z^{0}$ boson decays to $\mu^{+} \mu^{-}$about $3 \%$ of the time, providing a powerful tool to test the VD with highly energetic particles in the simplest topology. The effective resolution is measured through the so called dimuon miss distance $(\Delta)$, defined as the algebraic sum of the impact parameters $(\varepsilon)$ with respect to the beam spot centre. The impact parameter is signed depending on whether an observer standing at the beam spot centre and facing the direction of the track sees it on his right or left hand side. The miss distance is insensitive to the actual interaction point position in the beam spot, which has a width $\left(\sigma_{x}=150 \mu \mathrm{m}\right.$, $\sigma_{y}=10 \mu \mathrm{m}$ in the plane transverse to the beams) dominating the single-track impact parameter measurement error. The miss distance distribution width is $\sigma_{\Delta}=30 \mu \mathrm{m}$ for selected events from the 1991 data. It corresponds to a track impact parameter resolution $\sigma_{\varepsilon}=\sigma_{\Delta} / \sqrt{2}=21 \mu \mathrm{m}$. Scaling $\sigma_{\varepsilon}$ to account for the extrapolation from the VD to the interaction point gives a VD single-point resolution of $8 \mu \mathrm{m}$.

The impact parameter distribution on $Z^{0}$ 


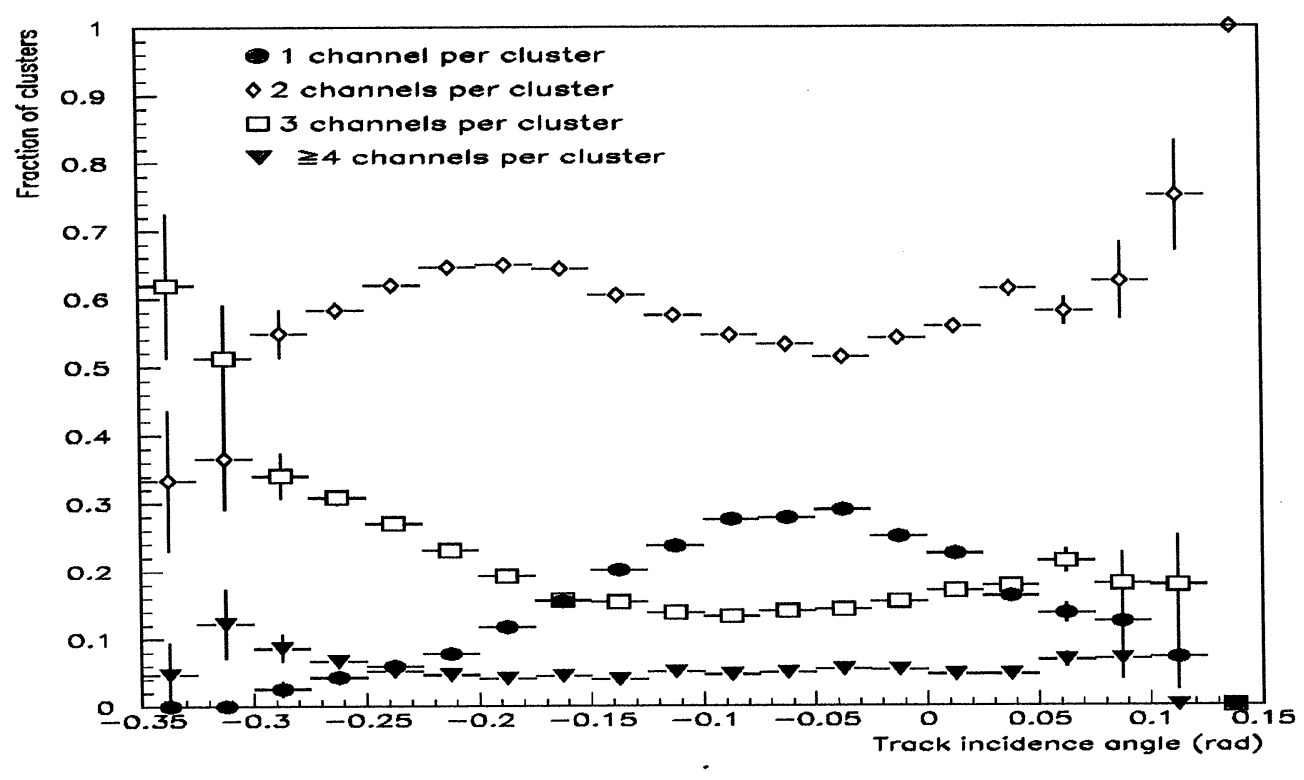

Figure 3. The fraction of clusters with 1,2,3 and more than 3 channels per cluster as a function of the incidence angle of a track in the silicon.

decays to multihadrons is the key to b lifetime measurements and heavy flavour tagging. The $\varepsilon$ resolution as a function of the projected momentum $\left(p_{t}\right)$ in the plane orthogonal to the beams is shown in Figure 4. Here $Z^{0}$ decays to light flavours have been selected (with approximate purity $83 \%$ and efficiency $42 \%$ according to Monte Carlo) and the impact parameter has been calculated with respect to the fitted primary vertex, in order to reduce the effect of the beam spot width. The measured curve can be parametrized as

$$
\sigma_{\varepsilon}=\frac{69}{p_{t}} \oplus 24 \mu m, p_{t} \text { in } \mathrm{GeV} / \mathrm{c}
$$

where the first term describes the multiple scattering contribution.

\section{Efficiency and misassociation}

Two detectors developed shorts during the installation and a few more had problems dur- ing the data taking period, resulting in $8.5 \%$ of the channels being dead at the end of 1991 . However, a $100 \%$ detector efficiency for 1 point and $93 \%$ for 2 points was maintained over the VD full acceptance.

In multihadron events, the average association efficiency of VD points to tracks reconstructed by the other DELPHI tracking chambers is $95.5 \%, 97.5 \%, 96.3 \%$ respectively for the Closer, Inner and Outer layers.

The ambiguity level in the pattern recognition is shown in Figure 5, where the fraction of tracks having a unique assignment of a VD set of points is displayed as a function of the number of tracks per half module. The distribution of maximum multiplicity per event within a half module is overlaid. In the class of tracks having 1 associated VD point, the ambiguity level increases from $16 \%$ for multiplicity 2 to $40 \%$ when 8 tracks cross the same half module. On the other hand, for tracks with 3 associ- 


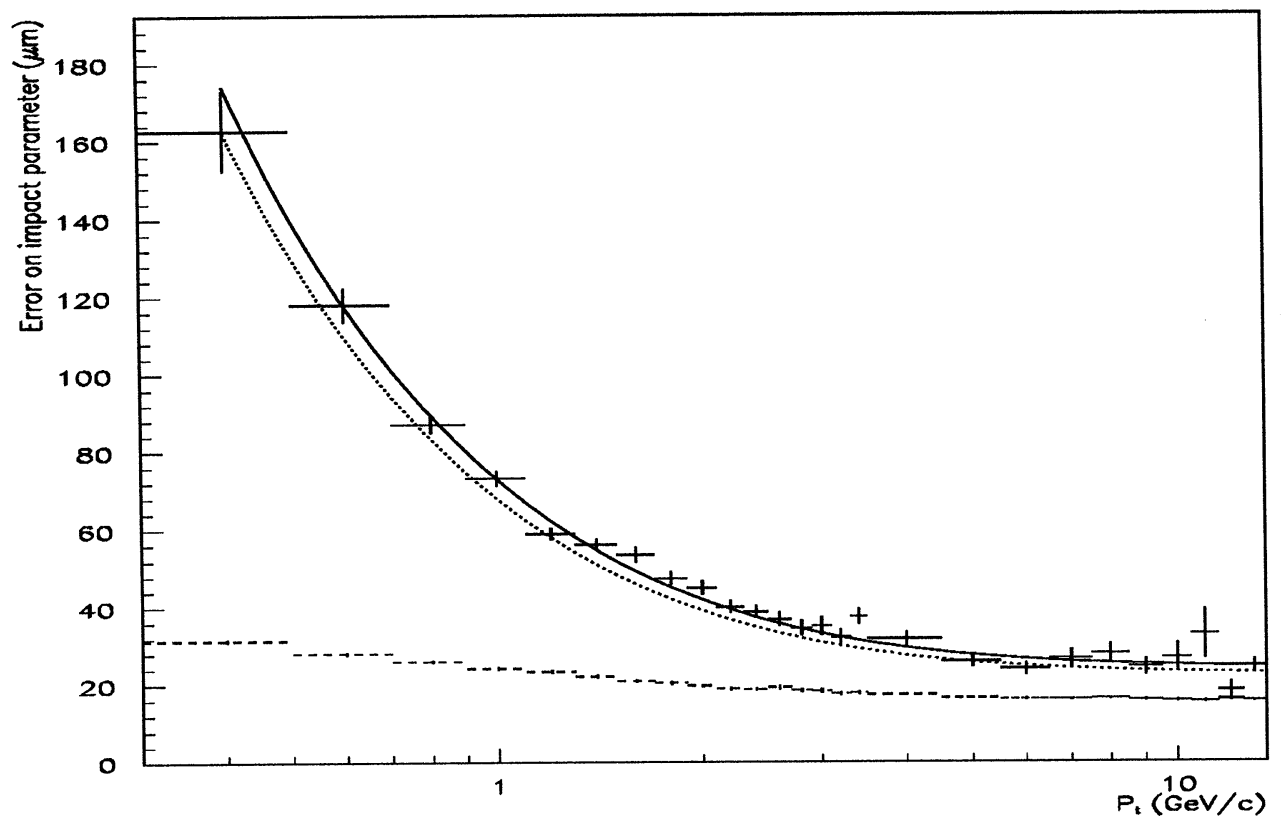

Figure 4. Impact parameter error versus $p_{t}$. The dashed data gives the contribution from the error on the vertex, which has been subtracted quadratically from the error per track.

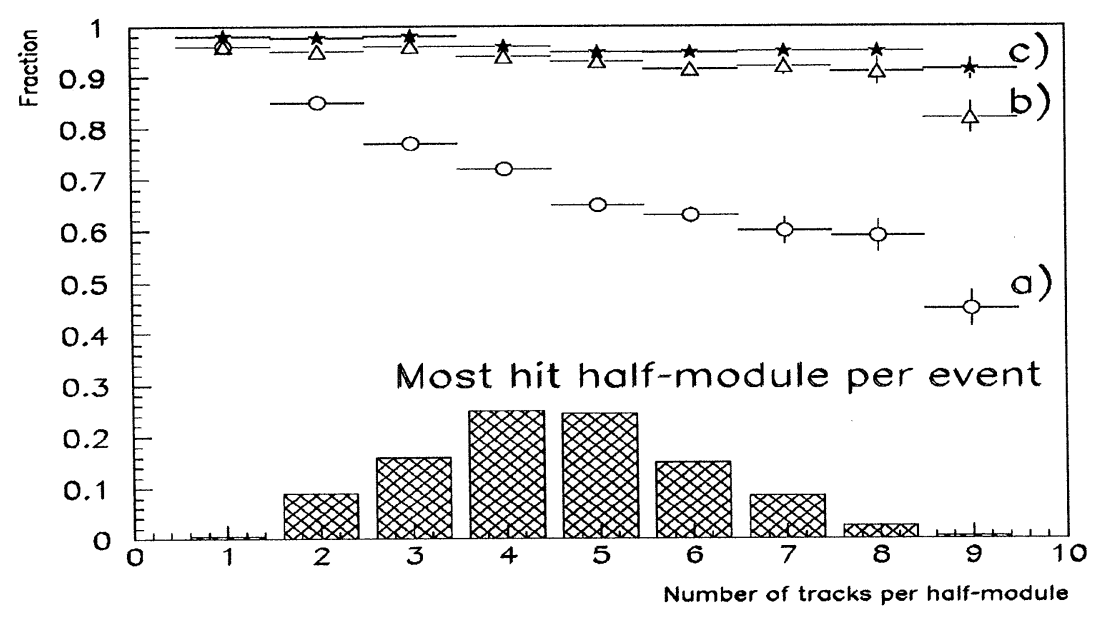

Figure 5. Fraction of tracks uniquely associated to clusters as a function of the track multiplicity for the cases of one silicon layer (a), two layers (b) and three layers (c). The distribution of maximum multiplicity within a half module is overlaid 
ated VD points the ambiguity level is reduced to $4 \%$ and it increases by $4 \%$ over the same multiplicity range. This result highlights the importance of a 3 layer geometry in the DELPHI environment beyond the pure single track reconstruction quality in simple topologies.

\section{CONCLUSIONS}

The Vertex Detector has been smoothly running in DELPHI since 1990 in the two layer configuration, upgraded to three layers in 1991. The design resolution has been achieved; it corresponds to a single point $8 \mu \mathrm{m}$ precision and an impact parameter resolution of $\sigma_{\varepsilon}=\frac{69}{p_{t}} \oplus 24 \mu \mathrm{m}$. The average association efficiency in multihadron events is $96 \%$. We plan to replace in 1993 the internal and external layers with double sided microstrip detectors, providing a precise information also along the beam direction. The three layer configuration will be mantained and the resolution in the transverse coordinate will not be degraded.

\section{REFERENCES}

1. - P. Abreu et al., DELPHI Collaboration, A measurement of the lifetime of the tau lepton, Phys. Lett. B267 pp. 422-430, (1991).

- P. Abreu et al., DELPHI Collaboration, Measurement of the average lifetime of B hadrons, Z. Phys. C Particles and Fields 53 pp. 567-580, (1992).

- P. Abreu et al., DELPHI Collaboration, Evidence for $B_{s}^{0}$ meson production at LEP, CERN PPE/92-104, submitted to Phys. Lett. B

- P. Abreu et al., DELPHI Collaboration, $\mathrm{A}$ measurement of $\mathrm{B}$ mesons production and lifetime using Dlepton events, DELPHI 92-78 PHYS 189
2. H. Borner et al., The DELPHI Microvertex Detector, DELPHI 92-92 PHYS 203, in preparation for Nucl. Instr. and. Meth.

3. J.C. Stanton, A Low Power, Low Noise Amplifier for a 128 Channel Detector Readout Chip, IEEE Trans. Nucl. Sci. 36, pp. 522-527, (1990)

4. M. Caccia et al., A Silicon strip detector with integrated coupling capacitor, Nucl. Instr. and Meth. A260, pp. 124-131, (1987).

5. P. Aspell et al, CMOS low noise amplifier for microstrip readout. Design and results, Nucl. Instr. and Meth. A301, pp. 506-516, (1991)

6. E. Belau et al., Charge collection in Silicon strip detectors, Nucl. Instr. and Meth. 214, pp. 253-260, (1983).

7. P. Weilhammer, Experience with Silicon Detectors in NA-32, CERN EP/86-54.

8. A. Andreazza, P. Biffi, C.Meroni, A. Stocchi, Results on the DELPHI Microvertex alignment from a precise 3-D mapping, Nucl. Instr. and Meth. A312, pp. 431-439, (1992).

9. R. McNulty, The Alignment of the DELPHI Microvertex Detector, DELPHI 92-40 TRACK 69, 27 March 1992.

10. M. Caccia et al., 1990 data taking period results of the DELPHI CDM System, Nucl. Instr. and Meth. A315, pp. 143-148, (1992). 